\title{
La Asociación Colombiana de Medicina Interna y la recertificación en medicina interna en Colombia
}

\author{
The Colombian Association of Internal Medicine \\ and recertification in internal medicine in Colombia
}

\author{
Alfredo Pinzón, Roberto D’Achiardı • Bogotá, D.C. (Colombia)
}

El ejercicio de la Medicina Interna se enfrenta a retos permanentes dado el entorno dinámico del cuidado de la salud, en el que los avances de las ciencias biomédicas deben integrarse racionalmente para proporcionar a la población una atención en salud coordinada y eficiente. Los numerosos desarrollos científicos y tecnológicos hacen de nuestra profesión médica una ciencia en cambio continuo, lo que exige al especialista la necesidad de mantenerse actualizado para procurar la mejor atención de sus pacientes.

Los estudios demuestran que en general, con el paso del tiempo el conocimiento médico se deteriora, y que los hábitos y patrones de la práctica médica fallan en cambiar en respuesta a los avances científicos (1). Uno de ellos concluyó que el tiempo transcurrido desde la última certificación del médico, se correlaciona con la disminución en la calidad de la atención de sus pacientes con hipertensión arterial. También se ha demostrado que los médicos que renuevan frecuentemente sus conocimientos y habilidades mediante metodologías estructuradas de mejoramiento de la calidad, logran mejor cuidado de sus pacientes, por ejemplo, una mejor calidad de atención a los pacientes con infarto agudo de miocardio se relacionó con disminución de mortalidad del 15\% (2-4). Análisis recientes indican que los médicos recertificados ofrecen a sus pacientes cuidados de salud de mayor calidad que quienes no lo están, y esto se correlaciona con mejor desempeño en un amplio rango de medidas de calidad, desenlaces favorables, atención más segura y mayores índices en actividades de prevención.

Si bien algunas habilidades y comportamientos del médico mejoran con el transcurso del tiempo (experiencia), también se sabe que existe una relación inversa entre el lapso desde la graduación y el cumplimiento de los estándares de calidad para el cuidado de los pacientes (5-7). Así mismo se sabe que los médicos no examinan rutinariamente y en forma independiente su propio desempeño y que su habilidad para hacerlo es limitada.

En la actualidad, la calidad en la atención es un derecho exigido por los pacientes y por nuestro sistema de salud, y la certificación de esa calidad mediante procesos de formación continua es un factor decisivo en la confianza y preferencias de los pacientes por sus médicos (8).

En cumplimiento de su objetivo estatutario: "Fomentar y mantener los más altos niveles de idoneidad y calidad conforme a las exigencias éticas en la formación y el ejercicio del especialista en medicina interna", la Asociación Colombiana de Medicina Interna $\left(\mathrm{ACMI}^{\circledR}\right)$ ha liderado desde hace más de 30 años el proceso de recertificación en Medicina Interna en Colombia, inicialmente bajo la forma pionera de Educación Médica Continuada .

Durante la presidencia del doctor Roberto Esguerra, en 1985 la ACMI ${ }^{\circledR}$ creó el Programa de Excelencia en Medicina Interna, basado en una tabla de créditos obtenidos por asistencia a eventos científicos, publicaciones, actividad docente, méritos académicos, conferencias dictadas y otros. Si el candidato cumplía con los requisitos establecidos según el análisis realizado por un comité conformado para tal fin, se le otorgaba bienalmente el Diploma de Excelencia en Medicina Interna.
Dr. Alfredo Pinzón Junca: Coordinador del Consejo de Acreditación y Recertificación (CAR) de la $\mathrm{ACMI}^{\circledast}$. Jefe Departamento de Medicina Interna Hospital Universitario de La Samaritana. Internista Hospital Simón Bolívar. Profesor Clínico Principal de Medicina Interna Universidad de La Sabana y Profesor Ad Honorem de Medicina Pontificia Universidad Javeriana; Dr. Roberto D'Achiardi Rey: Secretario del Consejo de Acreditación Recertificación (CAR) de la $\mathrm{ACMI}^{\circledast}$. Nefrólogo Fundación Cardioinfantil y Fundación Clínica Shaio. Nefrólogo Unidad Renal RTS - Cardioinfantil. Profesor Titular de Medicina Pontificia Universidad Javeriana y Universidad del Rosario, Profesor Clínico Principal Universidad de La Sabana. Bogotá, D.C. (Colombia).

Correspondencia. Dr. Roberto D'Achiardi Rey. Bogotá, D.C. (Colombia).

E-mail: rodachi@gmail.com

Recibido: 23/VI/2015 Aceptado: 25/VI/2015 
En 1990, siendo presidente el doctor Javier Molina, se estableció el Examen Bienal de Recertificación, basado en el MKSAP del American College of Physicians y se creó el Comité de Educación de la ACMI ${ }^{\circledR}$, con la participación de representantes de los departamentos de Medicina Interna de la Universidad Nacional, Pontificia Universidad Javeriana, Universidad de Caldas y Universidad Industrial de Santander, quienes colaboraron activamente en el desarrollo del programa.

A partir de 1998 se desarrolló el Curso Taller Presencial Bienal como preparación para el examen de conocimientos, que en 2002, en la presidencia del doctor Gustavo Márquez, fue cambiado a un curso y examen virtuales, infortunadamente de corta vigencia. No obstante, durante el primer decenio del siglo XXI se continuó con la certificación de Excelencia en Medicina Interna, con base en el análisis de la actividad académica y docente del internista, valorada por un comité asignado por la Junta Directiva de la $\mathrm{ACMI}^{\circledR}$ que debía incluir al menos un ex presidente de la asociación.

Luego se estableció el premio anual Beca ACMI ${ }^{\circledR}$-AstraZéneca de Excelencia en Medicina Interna, que estableció las categorías: internista docente/investigador, internista en práctica clínica, miembro asociado y miembro adherente, patrocinado por la industria farmacéutica aunque era de manejo independiente y exclusivo de la $\mathrm{ACMI}^{\circledR}$. Para su otorgamiento se consideraban criterios como el curso de renovación de conocimientos, conferencias dictadas, asistencia a reuniones o convocatorias generales, capacitaciones, asistencia al curso/taller nacional o capitular, trabajos de investigación, publicaciones, asistencia a eventos científicos, trabajos docentes o administrativos de trayectoria y proyectos e iniciativas coordinadas por la $\mathrm{ACMI}^{\circledR}$ y se entregaba como premio en cada categoría una generosa beca para asistir a eventos científicos o hacer rotaciones o estudios dentro o fuera del país.

Como queda claro, durante las últimas tres décadas, la $\mathrm{ACMI}^{\circledR}$ se ha esforzado constantemente por incentivar en sus miembros la conciencia de lo que hoy entendemos por recertificación médica voluntaria: mantenerse actualizado en los avances científicos de la especialidad, participar en eventos académicos, investigar y publicar, transmitir los conocimientos y habilidades, y presentar pruebas para evaluar la suficiencia de conocimientos cuando esto fuere necesario, para que según un sistema de puntaje establecido, el especialista pueda ser galardonado con un certificado de Excelencia en Medicina Interna que lo distinga como profesional de calidad superior.

Manteniendo la iniciativa de sus predecesores, en abril de 2012 el entonces presidente doctor Juan Manuel Senior solicitó al Comité de Educación y Comunicaciones de la $\mathrm{ACMI}^{\circledR}$ realizar una propuesta para establecer oficialmente un Programa de Recertificación en Medicina Interna. Ese comité, conformado por los doctores Juan Carlos Velásquez (Coordinador), Paulo Emilio Archila, Enrique Ardila, Análida Pinilla, Mario Fernando Escobar y Alfredo Pinzón, realizó un concienzudo estudio sobre la situación de la recertificación médica en Colombia y el mundo, generó un documento que recogió los antecedentes y desarrollo de este proceso, y propuso un detallado programa de recertificación en Medicina Interna, que de hecho fue la base para la cristalización de este anhelado proyecto.

En dicho documento se relata como la legislación colombiana había intentado establecer la Ley del Talento Humano en Salud (Ley 1164 de 2007), cuyo Artículo 10 delegaba a los Colegios Profesionales para: "recertificar la idoneidad del personal de salud con educación superior, de conformidad con la reglamentación expedida por el Ministerio de la Protección Social", y en su artículo 25 establecía que "para garantizar la idoneidad permanente de los egresados de los programas de educación en salud, habrá un proceso de recertificación como mecanismo para garantizar el cumplimiento de los criterios de calidad del personal en los servicios de salud"; y en su fallido intento de reglamentación consideraba criterios como: educación continuada (instituciones educativas), actividad docente, participación en investigación, tiempo de servicio, premios o reconocimientos, exámenes (comisión intersectorial) y evaluación del desempeño y competencias. Luego la Sentencia 756 de 2008 de la Corte Constitucional consideró que "la recertificación regula, restringe y limita el núcleo del ejercicio de la profesión médica al talento humano del área de la salud en todas sus manifestaciones, tanto como profesión, como ocupación, cambiando el paradigma de la competencia e idoneidad de los egresados de los programas de educación en salud antes y después de su vigencia, afectando el derecho fundamental en aspectos principales e importantes..." y en consecuencia declaró inexequible la totalidad del artículo 25 y el literal (d) del artículo 10 , con lo cual dejó un vacío legal en lo referente a la recertificación médica en nuestro país, que aún persiste y es tema de intenso debate.

Para consolidar este proyecto de recertificación en Medicina Interna, en diciembre de 2012 la presidenta de la $\mathrm{ACMI}^{\circledR}$, doctora Natalia Londoño, inscribió a nuestra asociación como miembro del Consejo Colombiano de Acreditación y Recertificación Médica de Especialistas y Profesiones Afines (CAMEC), que había sido recientemente creado bajo el liderazgo de la Asociación Colombiana de Sociedades Científicas como una organización sin ánimo de lucro, legítima y autónoma, encargada de congregar, homogeneizar, estandarizar y evaluar los procesos de certificación y recertificación de las asociaciones y sociedades científicas que lo componen (veintisiete a la fecha). Así mismo, en febrero de 2013 se creó el Consejo de Acreditación y Recertificación (CAR) de la $\mathrm{ACMI}^{\circledR}$, designando al doctor Alfredo Pinzón como su Coordinador (Presidente), quien en virtud de su cargo ha venido representando a la $\mathrm{ACMI}^{\circledR}$ ante el CAMEC y ha continuado impulsando el desarrollo y difusión de este necesario Programa de Recertificación, ahora con el irrestricto apoyo del actual presidente de la 
ACMI ${ }^{\circledR}$, doctor Helí Hernández, quien además gestionó la inclusión oficial del CAR en los recientemente reformados Estatutos de nuestra asociación.

Desde el 22 de noviembre de 2014 el CAR se encuentra formalmente constituido por un Consejo Superior conformado por el doctor Alfredo Pinzón (Coordinador) y un representante de cada capítulo de la $\mathrm{ACMI}^{\circledR}$ designado por su respectiva junta capitular: doctores Hubert Bahamón (Alto Magdalena), Óscar Osio (Antioquia-Chocó), Abelardo Montenegro (Caldas), Carlos Arturo Barrera (Caribe), Roberto D'Achiardi (Central), Ariel Bello (Costa Atlántica), José Porto (Morrosquillo), Álvaro Portilla (Nariño), Álvaro Granados (Norte de Santander), Arles Alarcón (Quindío), Mauricio Cárdenas (Risaralda) y Guillermo Salguero (Valle del Cauca). Para agilizar su funcionamiento, internamente se eligió una Junta Directiva, que quedó integrada así: presidente: doctor Alfredo Pinzón, secretario: doctor Roberto D'Achiardi y tres vocales, los doctores Ariel Bello, Álvaro Portilla y Guillermo Salguero. Además se creó el Comité de Exámenes liderado por el doctor Ariel Bello, con la participación de los doctores Abelardo Montenegro, Carlos Barrera,Arles Alarcón y Mauricio Cárdenas, quienes deben desarrollar y evaluar las futuras pruebas de suficiencia de conocimientos para la recertificación.

Tras arduo trabajo, el CAR desarrolló completamente el Programa de Acreditación y Recertificación en Medicina Interna (PARMI) de la ACMI $^{\circledR}$ (avalado por el CAMEC), que otorga la Recertificación Médica Voluntaria (RCMV) a los internistas en Colombia que cumplan con los requisitos que permiten distinguirlos como especialistas con desempeño profesional superior. Los contenidos, términos y requisitos de ingreso de este programa pueden ser consultados en nuestra página web:www.acmi.org.co

La RCMV en Medicina Interna que de hecho es voluntaria, es realizada y evaluada por pares académicos, no está restringida exclusivamente a los miembros de la $\mathrm{ACMI}^{\circledR} \mathrm{y}$ puede obtenerse como participante por primera vez o en forma iterativa. Tiene una vigencia de cinco años (quinquenio), y para efectos del primer ciclo regular, se aceptarán las actividades realizadas a partir del 1 de enero de 2014. Para obtener la RCMV el internista debe acumular mínimo 1000 puntos PARMI durante el periodo de cinco años para el que se haya inscrito, 500 de ellos (100 anuales) por Ejercicio Profesional Certificado (requisito sine qua non), y los otros 500 (mínimo 100 por año) por Actividades de Desarrollo Profesional, que incluyen educación médica continuada, desarrollo académico, logros profesionales, investigación y publicaciones, docencia universitaria, participación gremial, suficiencia demostrada mediante exámenes, o combinaciones de las anteriores. Si completa los requisitos del progra- ma, al finalizar el quinquenio el participante podrá obtener su Certificado y Diploma de Recertificación Médica Voluntaria en Medicina Interna expedidos por la $\mathrm{ACMI}^{\circledR}$ y el CAMEC, válida por los cinco años siguientes. Adicionalmente, si el participante obtiene más de 1000 puntos en el periodo por sus Actividades de Desarrollo Profesional, se hará acreedor a la Recertificación Médica Voluntaria con Excelencia.

Debe enfatizarse que la Recertificación Médica Voluntaria es un valioso aporte de los médicos al mejoramiento del sistema de atención en salud, que busca optimizar la calidad profesional desarrollando procesos estructurados de educación médica continua y actualización permanente de los especialistas, haciendo uso de los principales atributos que el sistema nos otorga como profesión: autonomía y autorregulación; en ningún momento se concibe como un acto obligatorio o punitivo que pretenda restringir el ejercicio médico (9).

En síntesis, tras 30 años de trabajo continuo de la ACMI ${ }^{\circledR}$ en recertificación voluntaria en la especialidad, en el pasado a través de la Certificación de Excelencia en Medicina Interna, hoy se ha dado un giro hacia la Recertificación Médica Voluntaria (RCMV) obtenida a través del Programa de Acreditación y Recertificación en Medicina Interna (PARMI), al cual se espera la masiva vinculación de los internistas colombianos (miembros o no de la $\mathrm{ACMI}^{\circledR}$ ).

La Junta Directiva Nacional y el CAR de la ACMI ${ }^{\circledR}$ se complacen en invitarlos a participar en el PARMI, que oficialmente abre sus puertas a todos los internistas en Colombia en una simbólica fecha para nuestra especialidad: 12 de julio de 2015: día del Internista.

\section{¡BIENVENIDOS!}

\section{Referencias}

1. American Board of Internal Medicine. Why Maintenance of Certification Matters. E105-09. 2012.

2. Holmboe ES, Wang Y, Meehan TP et al. Association between maintenance of certification examination scores and quality of care for medicare beneficiaries. Arch Intern Med 2008; 168: 1396-1403.

3. Norcini JJ, Kimball HR, Lipner RS. Certification and specialization: do the matter in the outcome of acute myocardial infarction? Acad Med 2000; 75: 1193-8.

4. Turchin A, Shubina M, Codos AH et al. Effect of board certification on antihypertensive treatment intensification in patients with diabetes mellitus. Circulation 2008; 117: 623-8.

5. Hartz AJ, Kuhn EM, Pulido J. Prestige of traininig programs and experience of bypass surgeons as factors in adjusted patient mortality rates. Med Care 1999; 37: 93-103.

6. Rhee SO. Factors determining the quality of physician's performance in patient care. Med Care 1976; 14: 733-50.

7. Choudhry NK, Fletcher RH, Soumeral SB. Systematic review: the relationship between clinical experience and quality of health care. Ann Intern Med 2005; 142: 260-73.

8. American Board of Medical Specialties. Facts about the 2008 ABMS consumer survey: How Americans choose their doctors (pdf). ABMS 2010.

9. Londoño N, Esguerra R. Evolución de la Medicina Interna en Colombia. Acta Med Colomb 2014; 39(4): 393-96. 